\title{
Clinical Predictors for Repeat Hospitalizations in Left Ventricular Assist Device (LVAD) Patients With Gastrointestinal Bleeding
}

\author{
Charles V. Welden ${ }^{\mathrm{a}, \mathrm{e}}$, Wiley Truss ${ }^{\mathrm{b}}$, Gerald McGwin ${ }^{\mathrm{c}}$, Frederick Weber ${ }^{\mathrm{d}}$, \\ Shajan Peter ${ }^{\mathrm{d}}$
}

\begin{abstract}
Background: Patients implanted with left ventricular assist devices (LVAD) carry an increased risk of gastrointestinal bleeding (GIB), estimated at $25 \%$ in most studies. Significant efforts are employed in localizing and stopping the source of bleeding, but the rates of repeat hospitalization for GIB remain surprisingly high. Given the increasing incidence of LVAD-dependent end-stage heart failure and the excessive costs associated with repetitive endoscopic investigations, risk factors associated with re-bleeding need to be determined. The aim of our study was to investigate clinical predictors associated with repeat hospitalizations for GIB in patients implanted with a LVAD.
\end{abstract}

Methods: We conducted a retrospective cohort using the prospectively assembled ventricular assist device database at the University of Alabama at Birmingham. We identified all end-stage heart failure patients who were implanted with a continuous-flow (CF) LVAD between Jan 1, 2009 and Dec 31, 2013. We excluded pulsatile devices, biventricular assist devices (BiVADs), right ventricular assist devices (RVADs), and patients under 19 years of age.

Results: There were 102 patients implanted with a CF-LVAD within the specified time period. With an average follow-up of 127 weeks, $32(31.4 \%)$ patients developed GIB requiring 79 separate hospitalizations. Average time from LVAD implantation to first bleed was 343 days. The re-bleeding rate requiring readmission was $56.3 \%$ in those admitted with GIB, with eight $(25 \%)$ of the patients necessitating multiple readmissions. The average hospital stay for a primary diagnosis of GIB was 9.45 days. Totally, 68 (86\%) patients required endoscopic evaluation during their hospitalization, with 35 (44\%) necessitating multiple procedures during the same admission. The

Manuscript submitted February 2, 2018, accepted March 1, 2018

aUniversity of Alabama School of Medicine, AL 35233, USA

bUniveristy of Alabama at Birmingham, Birmingham, Al 35203, USA

'Department of Epidemiology, University of Alabama at Birmingham, Birmingham, Al 35294, USA

dDepartment of Gastroenterology and Hepatology, University of Alabama at Birmingham, Birmingham, Al 35233, USA

${ }^{\mathrm{e}}$ Corresponding Author: Charles V. Welden, University of Alabama School of Medicine, Birmingham, AL 35233, USA. Email: charleswelden@uabmc.edu

doi: https://doi.org/10.14740/gr972w average time to first endoscopy was 2.5 days with a median of 2 days. Patients receiving early endoscopy ( $<48 \mathrm{~h}$ from admission) were $57 \%$ less likely to require future readmission for GIB compared to patients undergoing late endoscopy ( $>48 \mathrm{~h}$ ) (OR: $0.43, \mathrm{CI}$ : 0.19 - 0.9). Other factors associated with repeat admissions for GIB included indication for LVAD (bridge to transplant had OR: 0.07, CI: 0.02 - 0.27), male gender (OR: 10.4, CI: 1.8 - 59), length of initial hospital stay (OR: 0.83, CI: 0.71 - 0.97), and INR on admission (OR: 3.6, CI: 1.46 - 8.8). Although not statistically significant, patients undergoing subsequent endoscopies during a single admission were $84 \%$ less likely to develop re-bleeding in the future (OR: $0.158, \mathrm{CI}$ : 0.025 - 1.02).

Conclusions: GIB in LVAD patients is a significant problem with high rates of readmission despite extensive endoscopic investigations and anticoagulant adjustments. Our experience revealed that early endoscopy, longer initial hospital stay, and better INR control were all associated with decreased rates of readmission for GIB in this population. These modifiable factors should be emphasized and addressed in the future to reduce the burdens associated with repeated hospitalizations.

Keywords: Gastrointestinal bleeding; Left ventricular assist device; LVAD associated bleeding; Acquired von Willebrand disease

\section{Introduction}

There are an estimated 5.7 million patients suffering from congestive heart failure in the United States [1]. Due to annual increases in this disease, therapies aimed at improving patient burden are regularly being developed and modified. Therapies range from intravenous inotropic medications (milrinone, dobutamine) to implantable devices such as right ventricular assist device (RVAD), biventricular assist device (BiVAD), and left ventricular assist devices (LVAD). The benefit of ventricular assist device (VAD) is to provide continuous inotropic support capable of delivering adequate end-organ perfusion in patients with end stage heart failure. LVADs can be implanted as destination therapy in patients with end-stage heart failure not suitable for heart transplant or as a bridge to transplant. Over the past decade, the utilization of LVADs has markedly increased. Per the sixth INTERMACS annual report, an estimated 9,372 
LVADs were implanted between June 2006 and December [2]. The primary reason for this rise is related to the growing use of advanced life-sustaining measures in all types of cardiac disease coupled with the persistently limited number of cardiac organs available for transplant. In short, more patients are surviving and living longer with significant heart disease than ever before.

There are currently two main types of LVADs: pulsatile and non-pulsatile, or continuous-flow LVADs. A study done at 38 centers comparing pulsatile LVADs to continuous-flow LVADs showed that continuous-flow LVADs have a significant reduction in risk of stroke and pump thrombosis as well as a 2-year survival benefit [3]. Because of these advantages, continuous-flow LVADs have all but replaced pulsatile-LVADs over the last decade. The switch to a continuous-flow device has not been all positive, with one marked disadvantage being the significant increased rate of gastrointestinal bleeding (GIB) with these devices. A meta-analysis by Draper et al found the incidence of GIB in patients with continuous-flow LVADs to be $23 \%$ [4] , compared to previous studies in pulsatile LVADs that found a GIB rate closer to $10 \%$ [5]. Crow et al performed study comparing risk of GIB between pulsatile and non-pulsatile devices and found the risk of GIB to be 6.8 events per 100 patient years for pulsatile vs. staggering 63.8 events per 100 patient years for continuous LVAD [6].

Several studies have examined the etiology of GIB in this population, and multiple hypotheses exist. First, it has been clearly documented that patients implanted with a LVAD have a marked decrease in the von Willebrand glycoprotein, causing a form of acquired von Willebrand factor (vWF) deficiency. Interestingly, this deficiency resolves after heart transplant and the removal of the LVAD [7]. Second, multiple studies have shown the increased rate of angioectasia formation in this patient population. In a 2011 study, $31 \%$ of LVAD patients with GIB were found to have angioectasias as the potential cause for their blood loss [8]. Finally, in order to avoid the devastating complications of pump thrombosis and embolic strokes associated with LVADs, all patient require life-long antiplatelet and anticoagulation medications. These medications alone will increase the risk of GI hemorrhage in any population. Other etiologies such as mucosal ischemia related to the loss of the cardiac pump have also been suggested as causes for the increased risk of GIB, but are less well defined.

Overall, with the increasing utilization of LVAD therapy in end-stage heart failure along with the aging population gastroenterologists in all settings are likely to be confronted with GIB in this patient population. The economic burden of these hospital admissions is substantial including intensive care beds as well as multi-specialty consult services. Importantly, the rate of recurrence of GIB in this population is substantial, contributing to the significant healthcare costs associated with this population. While there have been multiple studies published regarding optimal approach to GIB in these patients, there have been very few examining the risk factors associated with the high recurrence of GIB. The aim of our study is to identify clinical factors associated with readmissions for recurrence of GIB in patients implanted with a LVAD.

\section{Methods}

\section{Patients}

We conducted a retrospective cohort study at a single tertiary care institute. The cohort included all patients implanted with a LVAD at the University of Alabama at Birmingham (UAB) between January 1, 2009 and December 31, 2013 who were admitted with a primary diagnosis of GIB. Patients with other devices such as BiVADs, RVADs, pulsatile devices and those less than 19 years of age were excluded. The aim of the study was to identify clinical predictors of repeat hospital admission for GIB in patients implanted with a LVAD.

\section{Endoscopy}

Throughout the specified time period, multiple endoscopic modalities were utilized in diagnostic and therapeutic approaches to the GIB. These included esophagogastroduodenoscopy (EGD), colonoscopy, push enteroscopy (PE), video capsule enteroscopy (VCE), as well as double-balloon enteroscopy (DBE). Each of these procedures has specific advantages and disadvantages ranging from time of procedure, ability to perform diagnostic and therapeutic interventions as well as percentage of bowel evaluated. The diagnostic and therapeutic yield of each procedure was documented along with the specified locations along the GI tract.

\section{Data collection}

The database was created and maintained on protected computers at the University of Alabama at Birmingham. Patients were followed until time data were collected in October 2015 and were evaluated for death or last follow-up at our tertiary care center. Both descriptive and clinical variables were collected including age, sex, race, indication for LVAD implantation, date and number of hospital admissions for GIB, length of hospital stay, INR on admission, number and type of procedures performed, antiplatelet/anticoagulation therapy used, diagnostic yield, therapeutic yield, location of lesion, and readmission rate. A total of 363 units of packed red blood cells (PRBCs) were given with an average of 5.11 per admission for GIB. Forty-five patients died after having LVAD but no reported deaths during an admission for GIB.

\section{Statistical analysis}

With the assistance of the Biostatistical Department at UAB, univariate analysis was performed on the data. Specifically, we used multivariable generalized estimating equation (GEE) models to test our hypothesis. A P-value $<0.05$ was considered statistically significant. We utilized SAS $₫$ version 9.3 for all of our statistical analysis. The dependent variable was repeat hospital admission for GIB. The independent variables included sex, race, overt vs. occult GIB, timing of endoscopy, diag- 
Table 1. Demographics

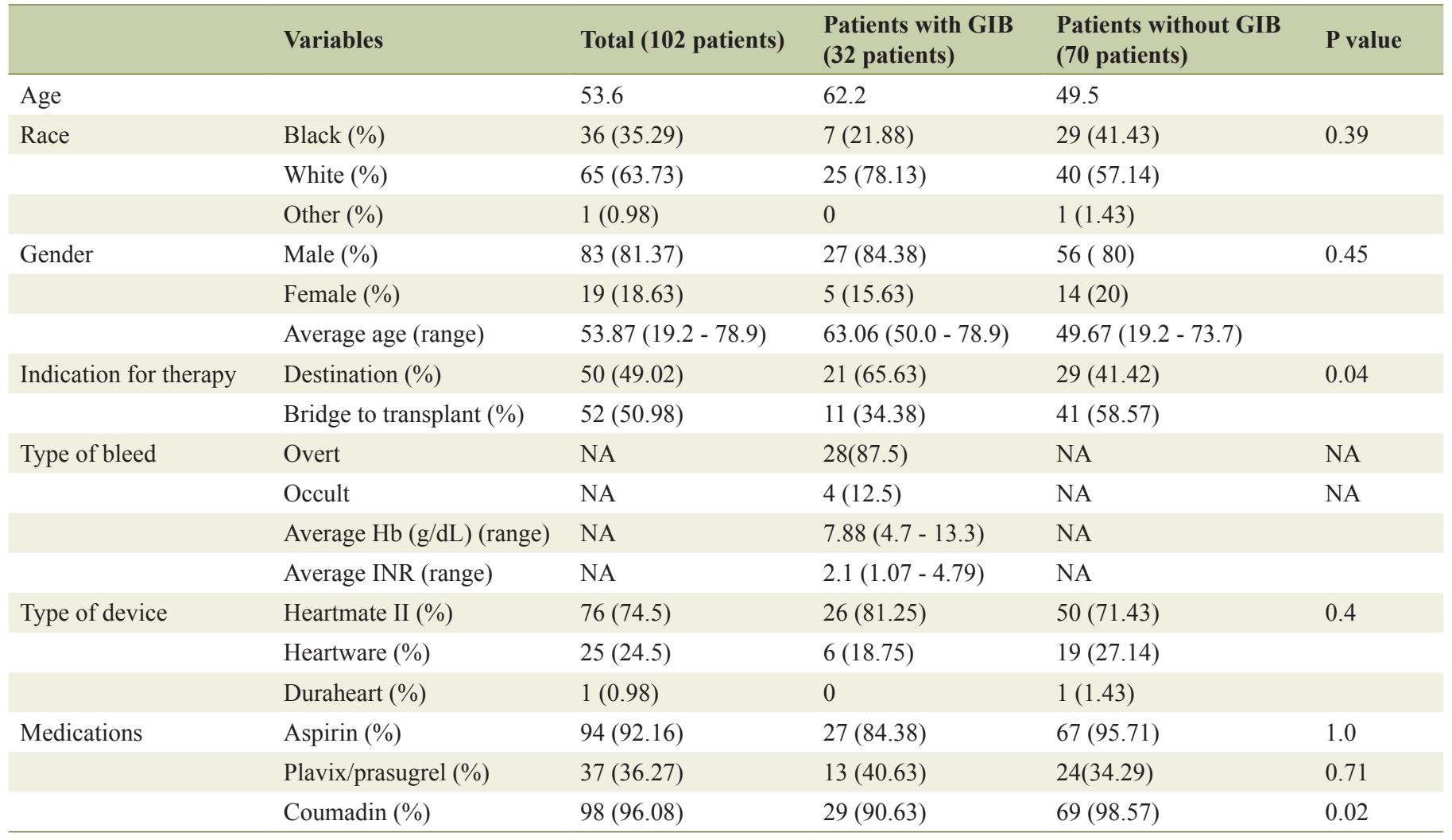

nostic yield, therapeutic yield, use of anticoagulation, use of antiplatelet medications, length of initial hospitalization, total units of transfusions, and total number of endoscopic procedures performed during initial hospitalization.

\section{Results}

\section{Patients}

There were 102 patients who received a LVAD at our institution between time period of January 2009 through December 2013. Table 1 shows the demographics of the patients included in the study. A total of 32 patients (31.4\%) required hospital admission for GIB and $18(56.3 \%)$ required repeat admissions. There were eight patients that had $>$ two hospitalizations for GIB. The average time from implantation of LVAD to presentation for GIB in our study was 343 days. Patient hospital length of stay for initial presentation of GIB was 9.45 days.

\section{Predictors of readmission}

Thirty one percent of our LVAD patients were admitted for a GIB and $56.3 \%$ of these required readmission and a total of eight $(25 \%)$ patients required $>$ two admission for GIB. Table 2 shows the factors that we identified which were predictive or

Table 2. Results Showing Odds Ratio (OR) for Readmission for GIB in LVAD Patients

\begin{tabular}{llllll}
\hline Parameter & Level & Estimated OR & Lower limit 95\% CI & Upper limit 95\% CI & P value \\
\hline Sex & Male & $10.369^{*}$ & 1.815 & 59.241 & $0.0085^{*}$ \\
Intention to treat & Bridge to transplant & $0.073^{*}$ & 0.19 & 0.273 & $0.0001^{*}$ \\
Overt vs. occult & Occult & 2.185 & 0.677 & 7.056 & 0.992 \\
Early endoscopy $(<48 \mathrm{~h})$ & Yes & $0.430^{*}$ & 0.186 & 1.254 & $0.048^{*}$ \\
Etiology & Ischemic & 0.571 & 0.260 & 0.979 & Not significant \\
LOS & Each additional day & $0.831^{*}$ & 0.712 & 8.759 & 0.092 \\
INR level on admission & Each 1 point over normal & $3.580^{*}$ & 1.463 & 0.052 & \\
\hline
\end{tabular}

LOS: length of hospital stay; INR: international normalized ratio; ${ }^{*}$ Showing statistically significant variables. 


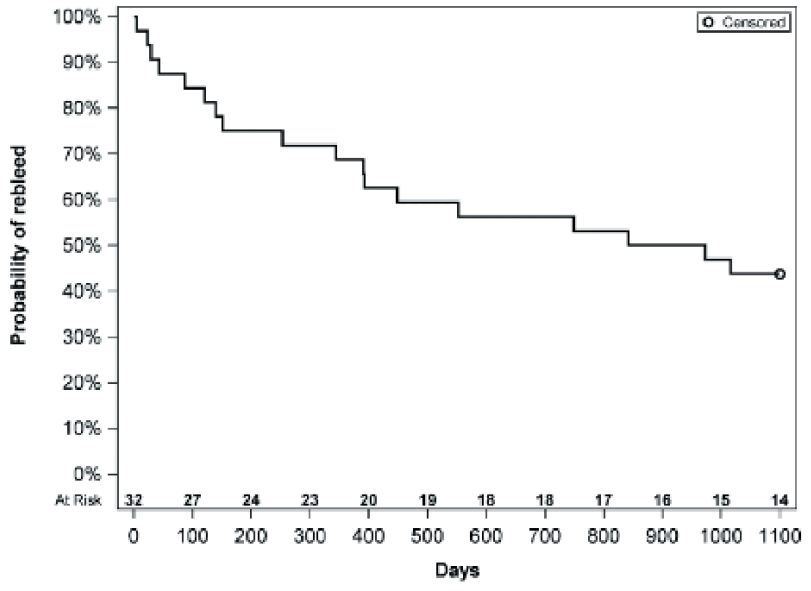

Figure 1. Kaplan-Meier curve showing "survival from re-bleeding".

protective of repeat admission. Male sex had an OR of 10.369 for repeat admission. Patients with supratherapeutic INR had an OR of 3.580 for every point the INR was over normal meaning that if patient had an INR of 3 (assuming normal is 1 ), they had $>7 \times$ risk of readmission. The more pertinent data are regarding those with OR $<1$ as these were protective of readmission for GIB. Patients who had LVAD as a bridge to transplant therapy as compared to destination therapy had the lowest OR at 0.073 . Patients who had early endoscopy as defined by $<48$ $\mathrm{h}$ from admission were shown to have readmission rate of $43 \%$ of those who had endoscopy $>48 \mathrm{~h}$ after admission. Lastly, for each additional day a patient stayed in hospital and had continued workup and management, this had a reduction of close to $17 \%$ readmission in future for GIB. Figure 1 is a Kaplan-Meier curve showing the survival from "rebleeding" in days from the initial bleeding event. It demonstrates that individuals "at risk" are more likely to re-bleed in the beginning as the slope of the curve starts to change around 400 days.

\section{Location of bleeding}

The location of bleeding was determined for each patient if able but $56 \%$ of patients were not able to have source localized initially but with repeat endoscopic evaluations, this number was reduced to $35 \%$ which correlated with $21 \%$ change. The most common site after all endoscopic workup was the jejunum at $21 \%$ (15\% was proximal) followed by colon at $13 \%$, duodenum and stomach at $9 \%$, ileum $6 \%$, esophagus $4 \%$, and lastly rectum at $3 \%$.

\section{Endoscopic evaluation}

Endoscopic evaluation for purposes of this study included EGD, colonoscopy, PE, DBE, VCE, and flexible sigmoidoscopy. Eighty-six percent of patients studied required endoscopic evaluation. The initial endoscopic evaluation study was dependent on patients presenting symptoms. The diagnostic yield comparing all forms of endoscopies in our study showed flexible sigmoidoscopy (Flex Sig) with 100\% diagnostic yield and a $67 \%$ therapeutic yield, with lowest being EGD which had 29\% diagnostic yield and 21\% therapeutic yield.

\section{Discussion}

The association between LVADs and GIB is evident through multiple studies. Our study showed a slightly higher risk of GIB at $31 \%$ as compared to Draper et al study of $23 \%$ [4]. Many patients are on triple antiplatelet/anticoagulation therapy for other cardiac diseases but the rates of GIB in those patients are much lower in the $1.5 \%-8 \%$ range [9]. So the question remains as to why patients with LVAD have such higher significant rates of GIB. Some of the proposed and published studies suggest that the use of antiplatelet/anticoagulation mediations, acquired von Willebrand disease, and development of AVMs are the main etiology and risk factors for development of GIB. Patients with LVADs have been shown to have lower levels of vWF after having an LVAD implemented with levels returning to baseline when device is removed $[7,10]$. Aortic stenosis is also felt to be a potential cause for reduction of $\mathrm{vWF}$ as the vWF undergo structural damage as it passes over the calcified valve which could be similar phenomena to when the vWF pass by the implanted LVAD [11]. The development of AVMs accounted for the source of GIB in $31 \%$ of the patients who had GIB associated with LVADs and occurred in older patients [8]. There are numerous studies, which were designed to create a best practice of evaluating this particular group of patients. Kushnir et al showed that with a comprehensive endoscopic evaluation the source of GIB can be identified in $70 \%$ of cases [12]. The diagnostic yield for our patients receiving PE vs. EGD was $42 \%$ vs. $29 \%$ respectively. The initial diagnostic endoscopic evaluation has also been studied and has been based on where culprit lesion is most commonly located. Draper et al developed an algorithm that recommends EGD and colonoscopy initially and if no lesion identified to pursue VCE. Based on VCE, patient either can go for deep enteroscopy of lesion identified or CT vs. MR enterography if no lesion identified on VCE [4]. A prior study at our institution looking at the use of VCE in LVAD patients showed that VCE was able to identify $67 \%$ of the bleeding source with jejunum identified in $78 \%$ and duodenum in $44 \%$ [13].

Not only do patients develop GIB, our study showed as much as $56.3 \%$ of these patients get re-admitted. America's healthcare costs continue to rise and ways to improve patient care while reducing the economic burdens on the system are always desirable. The important factors we identified in our study are the modifiable factors which include early endoscopy, length of stay, and INR level. Even though sex and use of LVAD as bridge to transplant are not modifiable, they still can help clinicians identify patients that are high risk and as such spend longer amount of time with them to help prevent repeat admissions. Why male patients are more likely to have readmission compared to females is not clear especially with article by Jahann and Shami showing that females with LVADs are more likely to have GIB [14]. One possible explanation to explain why those who have an LVAD as bridge to transplant 
might be protective could be related to their overall prognosis and self-awareness of their disease. If they are being considered for transplant, they must meet certain criteria and perhaps they are baseline "healthier" and have more motivation to follow the recommendations from their physicians. Although each additional day a patient stays in the hospital cost significant amount of money, there is a reduction of $17 \%$ for repeat admission which would easily justify the extra days spent at the hospital. When patients present with GI related complaints, emergency and gastrointestinal staff have to determine which patients required "emergent" therapy for complications such as GI bleed, biliary obstruction, food impaction, etc. Although GI bleed in patients is often not an "emergent" situation per se and can be managed initially with blood products and fluids, this study does suggest that early intervention (as defined by $<$ $48 \mathrm{~h}$ ) did indeed help reduce chance of repeat admissions for a GIB. Early endoscopy in setting of GIB increases the chance of a clinician finding culprit lesion and as a result allows them to have therapeutic intervention earlier. By waiting $>48 \mathrm{~h}$, there is a risk that the culprit lesion might have no stigmata of bleeding and as such will not be identified or therapeutically managed leading to a $57 \%$ increase risk of repeat admission for bleeding.

The clinical relevance of our study can boil down to two significant takeaways: patient morbidity and healthcare associated cost. A recently published study looking at cost and mortality showed that patients with continuous-flow LVADs related GIBs were found to have longer hospitalizations and higher hospital charges without a significant association with mortality [15]. This translates into more money being spent without any major benefit. The question remains as to how hospitals and clinicians can help and be proactive in reducing repeat admissions in these particular patients.

\section{Limitations}

One of the limitations of the study was the patient size. Despite being $>100$ patients, a larger study should be performed to confirm the risk factors that we identified as well as potentially identify additional factors that we did not include in this study. A second limitation is that this study occurred at only one tertiary care center. By combining additional tertiary care centers and having larger patient database, the chance of data having biases and confounding variables would be reduced.

\section{Conclusions}

With the ever increasing number of patients developed endstage systolic heart failure and needing LVADs as either bridge to transplant or destination therapy, the risk of GIB will continue due to patients requiring blood thinning medications. With an estimated $23-30 \%$ of patients with LVAD developing some form of GIB, a way to predict and more important prevent readmissions needs to be developed. Our study looked at this particular patient population and developed certain clinical predictors of readmission for repeat GIB. Our study found that male gender and degree of supratherapeutic INR levels were associated with higher admission rates whereas early endoscopy, longer stay in hospital and LVAD being used as a bridge to transplant were associated with lower readmission rates for GIB.

\section{Conflict of Interest}

The authors of this manuscript declare that they have no conflict of interest.

\section{References}

1. Writing Group M, Mozaffarian D, Benjamin EJ, Go AS, Arnett DK, Blaha MJ, Cushman M, et al. Heart disease and stroke statistics-2016 update: a report from the American Heart Association. Circulation. 2016;133(4):e38360.

2. Kirklin JK, Naftel DC, Pagani FD, Kormos RL, Stevenson LW, Blume ED, Miller MA, et al. Sixth INTERMACS annual report: a 10,000-patient database. J Heart Lung Transplant. 2014;33(6):555-564.

3. Slaughter MS, Rogers JG, Milano CA, Russell SD, Conte JV, Feldman D, Sun B, et al. Advanced heart failure treated with continuous-flow left ventricular assist device. N Engl J Med. 2009;361(23):2241-2251.

4. Draper KV, Huang RJ, Gerson LB. GI bleeding in patients with continuous-flow left ventricular assist devices: a systematic review and meta-analysis. Gastrointest Endosc. 2014;80(3):435-446 e431.

5. Guha A, Eshelbrenner CL, Richards DM, Monsour HP, Jr. Gastrointestinal bleeding after continuous-flow left ventricular device implantation: review of pathophysiology and management. Methodist Debakey Cardiovasc J. 2015;11(1):24-27.

6. Crow S, John R, Boyle A, Shumway S, Liao K, ColvinAdams M, Toninato $\mathrm{C}$, et al. Gastrointestinal bleeding rates in recipients of nonpulsatile and pulsatile left ventricular assist devices. J Thorac Cardiovasc Surg. 2009;137(1):208-215.

7. Meyer AL, Malehsa D, Budde U, Bara C, Haverich A, Strueber M. Acquired von Willebrand syndrome in patients with a centrifugal or axial continuous flow left ventricular assist device. JACC Heart Fail. 2014;2(2):141-145.

8. Demirozu ZT, Radovancevic R, Hochman LF, Gregoric ID, Letsou GV, Kar B, Bogaev RC, et al. Arteriovenous malformation and gastrointestinal bleeding in patients with the HeartMate II left ventricular assist device. J Heart Lung Transplant. 2011;30(8):849-853.

9. Barada K, Abdul-Baki H, El H, II, Hashash JG, Green $\mathrm{PH}$. Gastrointestinal bleeding in the setting of anticoagulation and antiplatelet therapy. J Clin Gastroenterol. 2009;43(1):5-12.

10. Aggarwal A, Pant R, Kumar S, Sharma P, Gallagher C, Tatooles AJ, Pappas PS, et al. Incidence and management of gastrointestinal bleeding with continuous flow assist devices. Ann Thorac Surg. 2012;93(5):1534-1540.

11. Sponga S, Nalli C, Charbonneau E. Severe upper gastro- 
intestinal bleeding in heartmate II induced by acquired von willebran deficiency: anticoagulation management. Annal Thoracic Surgery. 2012;94(2):e41-e43.

12. Kushnir VM, Sharma S, Ewald GA, Seccombe J, Novak E, Wang IW, Joseph SM, et al. Evaluation of GI bleeding after implantation of left ventricular assist device. Gastrointest Endosc. 2012;75(5):973-979.

13. Truss WD, Weber F, Pamboukian SV, Tripathi A, Peter S. Early implementation of video capsule enteroscopy in patients with left ventricular assist devices and obscure gastrointestinal bleeding. ASAIO J. 2016;62(1):40-45.

14. Jahann DA, Shami VM. Gastrointestinal bleeding during index hospitalization for mechanical circulatory support devices implantation: is the squeeze worth the ooze? Dig Dis Sci. 2017;62(1):7-9.

15. Li F, Hinton A, Chen A, Mehta NK, Eldika S, Zhang $\mathrm{C}$, Hussan $\mathrm{H}$, et al. Left ventricular assist devices impact hospital resource utilization without affecting patient mortality in gastrointestinal bleeding. Dig Dis Sci. 2017;62(1):150-160. 